\title{
PRINCIPLES FOR TIMING AT SPALLATION NEUTRON SOURCES BASED ON DEVELOPMENTS AT LANSCE
}

\author{
R. Nelson, R. Merl, and C. Rose, LANL, Los Alamos, NM 87544, USA
}

\begin{abstract}
Due to AC-power-grid frequency fluctuations, the designers for accelerator-based spallation-neutron facilities have worked to optimize the conflicting demands of accelerator and neutron chopper performance. For the first time, we are able to quantitatively access the tradeoffs between these two constraints and design or upgrade a facility to optimize total system performance using powerful new simulation techniques. We have modeled timing systems that integrate chopper controllers and chopper hardware and built new systems. Thus, at LANSCE, we now operate multiple chopper systems and the accelerator as simple slaves to a single master-timingreference generator. Based on this experience we recommend that spallation neutron sources adhere to three principles. First, timing for pulsed sources should be planned starting with extraction at a fixed phase and working backwards toward the leading edge of the beam pulse. Second, accelerator triggers and storage ring extraction commands from neutron choppers offer only marginal benefits to accelerator-based spallation sources. Third, the storage-ring RF should be phase synchronized with neutron choppers to provide extraction without the one orbit timing uncertainty.
\end{abstract}

\section{INTRODUCTION}

Pulsed spallation neutron sources feature the production of neutrons at approximately the moment the proton beam strikes the target. The details of this approximation depend upon the particular complexities of the materials and geometry of the target as well as the time profile of the incident beam. Our interest is not with these details, but rather the obvious fact that this neutron production follows the arrival of the beam on target, and for neutron sources with storage devices, this moment is defined by the extraction timing. We will restrict our discussion to neutron sources of this type.

Many neutron scattering instruments wish to tailor the neutron energy incident on the sample. Often this filtering is nothing more than removing the undesired high-energy neutrons that end up as background events if allowed entry to the instrument. Sometimes the range of energies must be limited to remove ambiguities encountered in the time-of-flight measurements. To explore inelastic phenomena some instruments desire a narrow energy band for the incident neutron.

Typically neutron energy filtering is accomplished through the use of energy-dependent reflections, absorption, or neutron choppers. We will consider only the choppers and the timing challenges common to operations with an accelerator and storage ring.

\section{PLAN THE ACCELERATOR TIMING FROM STORAGE RING EXTRACTION}

From the perspective of the neutron scattering instrument the experiment begins when the beam pulse strikes the target. The clocks in the data acquisition system start the measurement of the interval during the flight time of the neutrons from the source through the sample to the detector. Any choppers in the instrument beamline are phased to trending algorithms established by the master timing generator for chopper controllers.

This orderly picture can be disrupted by any unexpected changes in the phase between beam extraction and the choppers. Presumably the phase of the choppers will be changed only in the event that the instrument wants to alter the energy content of the beam on the sample. Unfortunately some existing accelerators like ours at LANSCE are designed to initiate acceleration of the beam at a fixed starting time with respect to the last zero crossing of the power grid. If these facilities are operated such that the length of the beam pulse loaded into the storage ring is used to adjust the maximum circulated beam intensity, then the trailing edge of the beam pulse usually defines the timing for extraction. In Figure 1 beam gates A and B illustrate this mode of operation. The time for extraction is marked with the "arrow" on the trailing edge of each beam gate. Thus the phase of extraction varies with the intensity of the stored beam. For the choppers, this change of phase is arbitrary and unexpected.

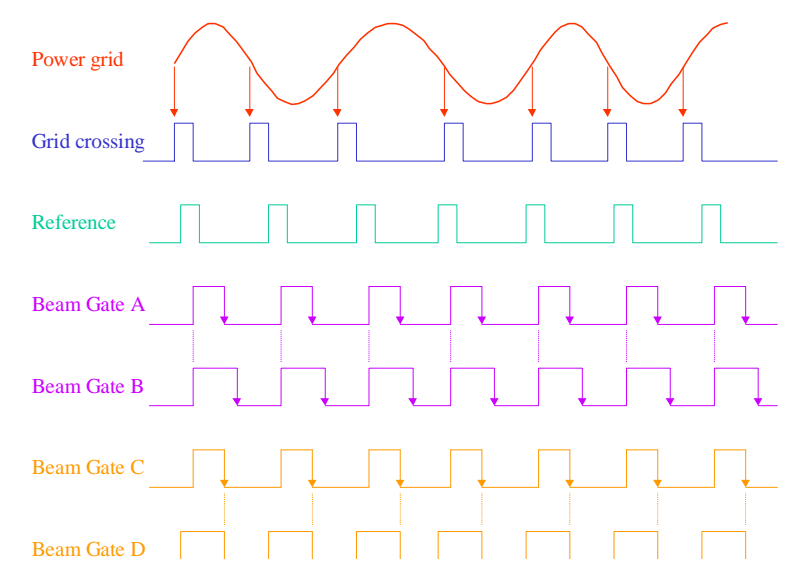

Figure 1. Relation of extraction to the timing reference signal. The accelerator may operate with fixed phase for the leading edge or the trailing edge of the beam as depicted with beam gates A-B or C-D, respectively. 
To eliminate phase changes accelerator and storage ring designers and operators should extract beam at a fixed time with respect to zero crossing of the power grid as beam gates $\mathrm{C}$ and $\mathrm{D}$ show in Figure 1. In effect the plan for timing should start with extraction and work backwards towards the "leading" edge of the pulse. If so structured, then the choppers follow only those phase changes due to the power grid fluctuations or due to the need for different experimental conditions.

\section{OMIT ACCELERATOR TRIGGER AND STORAGE RING EXTRACTION}

Spallation sources in the United States (LANSCE, IPNS, and SNS) operate or will operate their accelerator systems synchronized with the power grid. In older facilities this constraint enabled designers to simplify the RF power modulators. Furthermore if locked to the grid, the facility eliminates any "beat frequency" noise in timeof-flight neutron events. In contrast with these advantages, the neutron choppers suffer operational disadvantages. Since the choppers consist of massive blades rotating in the neutron beam and lethargic motors, they can not easily accommodate the phase changes present in the power grid.

To mitigate the conflicting demands of the accelerators and choppers, designers developed elaborate control systems at LANL [1,2] and ANL [3,4]. Figure 2 illustrates the block diagram for the architecture of these timing systems. The power grid drives the timing reference generator that performs the tradeoffs between accelerator and chopper performance. The resultant timing signal then drives the accelerator and the master neutron chopper. Since the master chopper is generally unable to follow the timing signal, the master chopper would trigger acceleration of the beam or extraction of the beam from a beam storage device or both. The resulting extraction signal is then used to synchronize the remaining choppers.

Like its peers in the US, the SNS initially adopted the "conventional" timing strategy illustrated in Figure 2. However, recent work at LANSCE has impacted this timing architecture and other timing concepts, and

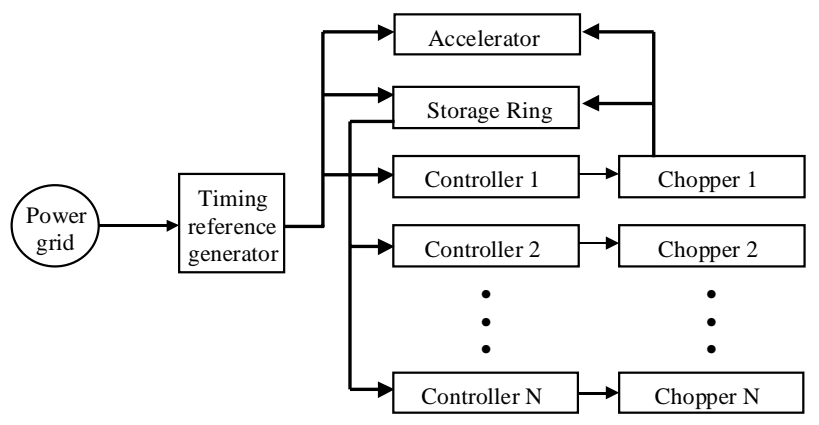

Figure 2. Block diagram for timing system dependencies. The master chopper, chopper 1 , triggers the accelerator and/or storage ring. The other choppers are phased with respect to ring extraction.

suggests revisions are appropriate. Our work focused on the timing reference generator [5] and the chopper controllers [6,7].

At LANSCE we have developed devices for the timingreference generator and chopper controllers. The accelerator operations staff developed a secondgeneration timing reference generator [2] that filtered the power grid variations as much as permitted by reliable accelerator performance. Since the accelerator has minimal beam losses when the filtered timing reference pulses are within $\pm 100 \mu$ s of the raw power grid, the timing generator maintains a close coupling to the grid. Subsequently the instrument support staff developed controllers for the Fermi and $\mathrm{T}_{0}$ choppers to exploit the chopper-friendly timing reference. The resulting performance as measured by the standard deviation of the interval between top-dead-center (TDC) and the timing reference pulse improved between one and two orders of magnitude.

With the benefit of simulation tools that enable modeling of the complete chopper system from power grid to rotating blade, we have developed a thirdgeneration timing reference generator. Since the smoothing algorithm executes in a digital signal processor, many filters are possible and we have implemented several. This device has a "control knob" that allows us to select how closely we chose to follow the grid. We find it convenient to characterize the coupling to the grid as the standard deviation of the interval between the zero crossing for the raw grid and the timing reference pulse. We define this quantity as the accelerator-tracking sigma. Similarly we define the chopper-tracking sigma as the standard deviation of the interval between the TDC of the chopper rotor and the timing reference pulse.

The "knob" enables us to explore chopper performance as a function of coupling to the grid. As can be seen from the data in Figure 3 taken with the third-generation timing reference generator, the $600-\mathrm{Hz}$ Fermi chopper performance increases dramatically as the acceleratortracking sigma rises above $3 \mu \mathrm{s}$. In Figure 3 we also present the performance for the $20-\mathrm{Hz} \mathrm{T}_{0}$ chopper. For

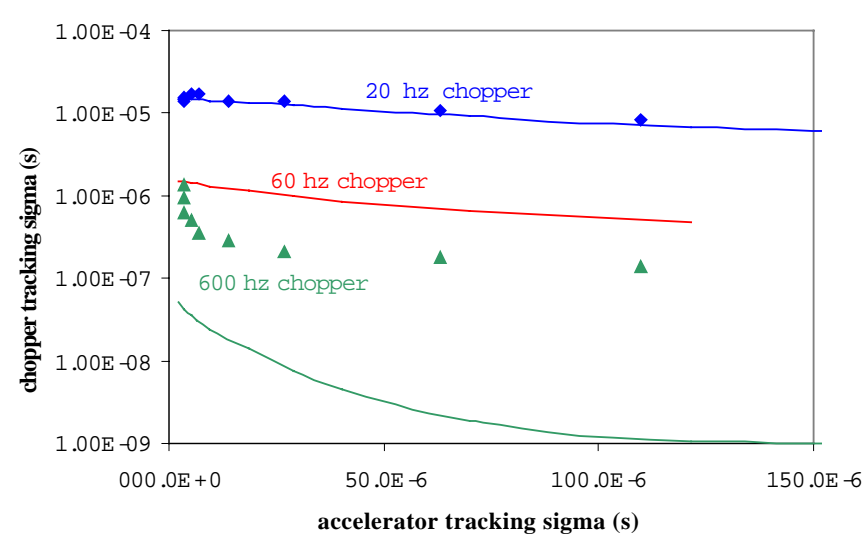

Figure 3. Predictions (lines) and measurements for chopper performance as a function of the coupling to the power grid. 
20- $\mathrm{Hz}$ data the measurements are in excellent agreement with simulations.

To compare the timing generators we measured the accelerator-tracking sigma for the second-generation timing generator at $25 \mu \mathrm{s}$. Then we set our "knob" to yield the same accelerator-tracking sigma. At this point we observed a chopper-tracking sigma of $200 \mathrm{~ns}$, down from $600 \mathrm{~ns}$ for the second-generation timing generator

Since the performance of choppers and chopper controllers is excellent without invoking complex interdependencies as shown in Figure 2, we now operate the LANSCE accelerator and storage ring as well as neutron choppers as slaves to the master timing reference generator. This allows a significant simplification as shown in Figure 4.

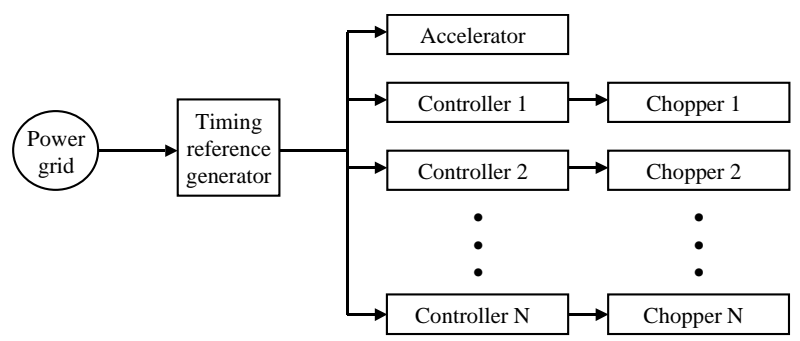

Figure 2. Block diagram for simplified timing-system dependencies. In contrast with Figure 2 the accelerator/storage ring and the chopper controllers all operate as slaves. No chopper enjoys a special status as the master chopper.

\section{SYNCHRONIZE THE RING RF AND THE CHOPPERS}

The storage ring for SNS has a circulation period that is dependent upon the orbit of the proton beam. Since the period may vary as much as $1 \mathrm{~ns}$, the time to load the ring with 1000 turns can vary by $1 \mu \mathrm{s}$, which is comparable to circulation period for a ring with a nominal frequency of $1.05 \mathrm{MHz}$. The accelerator-control timing can accommodate this variable frequency by distributing the ring $\mathrm{RF}$ to all timing modules and measuring all timing in coarse units of ring RF cycles and in fine units of absolute time for intervals less than the ring period [8]. Initially the SNS timing plans called for the chopper to request extraction during a window approximately $10 \mu \mathrm{s}$ long. Beam would be extracted on the next orbit leaving a $\pm 0.5 \mu$ s timing uncertainty.

We see an opportunity to eliminate this uncertainty. If the acceleration, storage and extraction of the beam are choreographed from the moment of extraction backward in time, then we know when to satisfy setup-timing requirements and when the accelerator must be prepared to launch the leading edge of the beam. This lead-time can be expressed as a multiple of ring RF cycles, and it will be a constant for a specific "mode" of the accelerator. So in advance of this lead-time, the timing generator can shift the phase of the fixed-frequency ring RF to synchronize with the timing-reference pulses. This phase change amounts to a modest shift of $\pm 0.5 \mu$ s or less that must preformed while the accelerator and ring are empty. In so doing the ring RF will automatically fall into synchronization with the choppers that are also slaved to the timing-reference pulses.

In response to our developments the SNS now plans to synchronize the ring RF with the master timing system approximately $6000 \mathrm{RF}$ cycles prior to extraction [9].

\section{CONCLUSIONS}

New spallation neutron sources should break with traditional timing architectures and:

- plan the accelerator timing from storage ring extraction

- omit accelerator triggers and storage ring extraction

- $\quad$ synchronize the storage ring RF and the choppers

In so doing the facilities will simultaneously simplify timing operations and minimize jitter that choppers must follow.

\section{REFERENCES}

[1] Bolie, V.W.; Brugger, R.M.; Silver, R.N., "Neutron chopper phasing for a quasi-periodic pulsed neutron source," Nuclear Instruments \& Methods in Physics Research, 1 May 1985; vol.A236, no.1, p.85-94.

[2] Rybarcyk, L.J.; Shelley, F.E., Jr, "Improvements to the LANSCE accelerator timing system," 1997 Particle Accelerator Conference, 12-16 May 1997, Vancouver, BC.

[3] Rauchas, A.; Ostrowski, G.; Pelizzari, C.; Volk, G., "IPNS accelerator system and neutron chopper synchronization," ICANS-VII. Proceedings of the Seventh Meeting of the International Collaboration on Advanced Neutron Sources (AECL-8488), 13-16 Sept. 1983, Chalk River, Ont., Canada.

[4] Ostrowski, G.E.; Donley, L.I.; Rauchas, A.V.; Volk, G.J.; Jung, E.A.; Haumann, J.R.; Pelizzari, C.A., "The PNS Chopper control and accelerator interface systems," Proceedings of the Eighth Meeting of the International Collaboration on Advanced Neturon Sources (RAL-85110), 8-12 July 1985, Oxford, UK.

[5] Rose, C., Lara, P., Merl, R., Nelson, R., "A TimingReference Generator for Power-Grid-Synchronized Neutron-Spallation Facilities," PAC 2001, Chicago, IL, US.

[6] Merl, R., Kupcho, K., Nelson, R. "Design and Performance of a DSP Based Neutron Chopper Phase Controller," PAC 2001, Chicago, IL, US.

[7] Rose, C., Lara, P., Merl, R., Nelson, R., "A Simple Method for Real-Time DSP-Based Neutron Chopper Speed and Phase Control," PAC 2001, Chicago, IL, US.

[8] "Spallation Neutron Source Systems Requirements Document For Timing System," SNS 109020000SR0001-R00, January 2000.

[9] private communication, May 2001. 